\title{
Associação entre o uso de antirretrovirais no tratamento para HIV e alterações físicas e metabólicas
}

Aurélio Goulart Rodovalho ${ }^{1}$; Franco Nero Tristão ${ }^{2}$; Laila Lidiane da Costa Galvão $^{3}$; Ricardo Goulart Rodovalho ${ }^{4}$; Richelle Cristine Torunsky ${ }^{5}$; Roselma Lucchese ${ }^{6}$

\section{Resumo}

O uso das terapias antirretrovirais é essencial para a expectativa de vida de quem vive com HIV/Aids. No entanto, estudos mostram que o uso dos múltiplos fármacos pode induzir a efeitos colaterais associados a distúrbios metabólicos. Dentre as alterações mais frequentes está a Síndrome Metabólica, que se caracteriza como um conjunto de fatores de riscos, relacionados entre si, com patogênese multifatorial e que predispõe ao desenvolvimento de hipertensão, diabetes mellitus e dislipidemias. Alterações no metabolismo ósseo também representam um importante evento associado ao tratamento para HIV/Aids. Estudos apontaram que pessoas em uso de antirretrovirais apresentaram maiores chances de sofrerem fraturas e ainda de desenvolver osteoporose. Esta revisão apresenta uma atualização dos fatores epidemiológicos e clínicos das complicações metabólicas associadas ao uso de antirretrovirais no curso da infecção pelo HIV. Contudo, a Aids deve ser encarada como uma doença que requer atenção, responsabilidades de assistência e cuidados específicos, o que exige uma rede profissional multidisciplinar capaz de monitorar e minimizar os efeitos colaterais associados a terapia antirretroviral.

Palavra-chave: HIV; síndrome metabólica

1 Biomédico, especialista em Gestão Hospitalar e mestrando em Gestão Organizacional pela Universidade Federal de Goiás. E-mail: aureliorodovalho@ hotmail.com. Catalão - Brasil

2 Médico Infectologista pela Universidade Federal de Goiás. E-mail: franconerotristao@bol.com.br. Catalão - Brasil

3 Administradora, mestranda em Gestão Organizacional pela Universidade Federal de Goiás.

E-mail: laila@iftm.edu.br. Patrocínio - Brasil

4 Biólogo, doutorando em Biotecnologia e Biodiversidade pela Rede Pró Centro-Oeste. Email: ricardogrodovalho@ hotmail.com. Goiânia-Brasil

5 Enfermeira pelo Centro Universit Franciscano. E-mail: richerct@ gmail.com. Catalao-Brasil

6 Enfermeira, doutora em Enfermagem pela Universidade de São Paulo. E-mail: roselmalucchese@ hotmail.com. Catalão - Brasil 


\section{INTRODUÇÃO}

A Síndrome da Imunodeficiência Adquirida (Aids) é uma severa imunossupressão associada com o surgimento de infecções oportunistas e alguns tipos de neoplasias, causada pelo Vírus da Imunodeficiência Humana (HIV) (COFFIN et al, 1986).

A infecção pelo HIV é uma pandemia, com diferenças sócio demográficas, considerada um dos principais problemas de saúde pública (VASIMON et al, 2011). Estimase que, atualmente, 35 milhões de pessoas estejam infectadas pelo HIV em todo mundo, dentre as quais, 7 milhões são crianças ou jovens com menos de 24 anos de idade (UNAIDS, 2014). No Brasil cerca de 734 mil pessoas vivem com HIV/Aids, correspondendo a uma prevalência de 0,4\% (MINISTÉRIO DA SAÚDE, 2014).

Do início da epidemia (1980) até dezembro de 2013, foram registrados 278.306 óbitos tendo como causa básica a Aids. As principais formas de transmissão do vírus ocorrem pela transferência de fluidos biológicos contaminados como: relações sexuais, compartilhamento de objetos perfurocortantes contaminados, e de mães infectadas com HIV na fase intra-uterina ou durante a amamentação (TSE et al, 2015).

A evolução da Terapia Antirretroviral Fortemente Ativa (HAART) e as políticas de acesso universal ao tratamento provocaram um impacto na história do HIV (PONTE et al, 2009), no qual inquestionáveis progressos foram conquistados, implementando estratégias para o acompanhamento e o tratamento dos portadores do vírus (LEITE, 2010). O melhor entendimento da patogenia, aliado a adesão do paciente ao tratamento, promoveu uma importante supressão no processo de replicação viral (VALENTE et al, 2005). Dessa forma, a adoção de combinações terapêuticas contendo diferentes classes de drogas proporcionou uma drástica redução na taxa de mortalidade e um aumento considerável na qualidade de vida das pessoas que vivem com HIV (PONTE et al, 2009; SIGNORINI et al, 2010).

No entanto, os efeitos colaterais associados à terapia vêm sendo descritos como fatores de risco para problemas crônicos (ALMEIDA et al, 2009). Inúmeros eventos estão relacionados a alterações psíquicas, endócrino-metabólicas e físicas que levam ao desenvolvimento de enfermidades como hipertensão, diabetes mellitus (PAPI et al, 2014), dislipidemias (GUEVARA et al, 2008), disfunções hormonais (PONTE et al, 2009), alterações hematológicas como anemia, leucopenia e trombocitopenia (KIBARU et al, 2015), nefropatias (ARAÚJO et al, 2007) e ainda manifestações psicossociais (REMOR, 2002).

Para o século XXI, vários estudos demonstram que o uso de HAART pode induzir a um processo de instalação de Síndrome Metabólica (SM) (ACOSTA et al, 2013; ALMEIDA et al, 2009; ARAÚJO et al, 2007; GUEVARA et al, 2008; SIGNORINI, et al, 2010; VALENTE et al, 2005; VASIMON, et al, 2011). A SM é um conjunto de fatores de risco, relacionados entre si, com patogênese multifatorial e que predispõe ao desenvolvimento de hipertensão, diabetes mellitus e dislipidemias (GUEVARA et al, 2008). As consequências clínicas para estas condições incluem aumento do risco de doenças cardiovasculares, consideradas atualmente como a principal causa de morte no mundo (GUEVARA et al, 2008; SIGNORINI et al, 2010). Dentre as alterações no metabolismo lipídico associadas ao tratamento com HAART, predomina a redução dos níveis séricos do HDL-Colesterol e aumento dos níveis de Colesterol total, LDL-Colesterol e Triglicerídeos, constituindo um perfil aterogênico (VALENTE et al, 2005). 
O uso das terapias antirretrovirais é essencial para a expectativa de vida de quem vive com HIV/Aids. TSE et al (2015), estudou a partir de uma premissa econômica a eficácia da introdução de HAART e identificou um considerável custo-benefício associado ao tratamento quando comparado a outros regimes terapêuticos. Entretanto, as estratégias adotadas para contenção dos efeitos secundários ao uso das drogas ainda não estão muito bem estabelecidas. Sendo assim, é fundamental rastrear e descrever produções científicas que ofereçam soluções frente a essa temática.

\section{SINDROME DA IMUNODEFICIÊNCIA ADQUIRIDA}

\subsection{Histórico}

A década de 80 foi marcada pelos primeiros relatos de uma nova patologia que viria a se tornar uma epidemia em escala global (BAZIN et al, 2014; COFFIN et al, 1986). Com os primeiros casos descritos em 1981, a infecção foi inicialmente identificada nos Estados Unidos, África Central e Haiti. Em 1983 a Síndrome da Imunodeficiência Adquirida (Aids), assim denominada por já se conhecer sua condição com um quadro de imunossupressão, foi associada a um retrovírus como causador da doença. No entanto, somente em 1986 usou-se a denominação de Vírus da Imunodeficiência Humana (HIV) para caracterizar o agente etiológico causador da doença (COFFIN et al, 1986).

Desde então, inúmeros avanços permitiram um futuro promissor no controle da epidemia. Dentre eles, o desenvolvimento e aperfeiçoamento de testes laboratoriais, cada vez mais precisos quanto aos padrões de sensibilidade e especificidade; a utilização de múltiplos fármacos antirretrovirais; e a criação de políticas públicas que possibilitaram implantar rotinas pautadas na integralidade do cuidado à saúde (BAZIN et al, 2014), são os fatores históricos de maior impacto.

No Brasil, o acesso universal e gratuito às medicações antirretrovirais foi implantado em 1996. Em 2013 um marco o colocou em evidência no cenário mundial, sendo o primeiro país em desenvolvimento e o terceiro do mundo a iniciar imediatamente o uso da terapia antirretroviral a todas as pessoas que viviam com HIV/Aids (MINISTÉRIO DA SAÚDE, 2014). Esta iniciativa visou principalmente aumentar a qualidade e expectativa de vida e reduzir a transmissão do HIV.

\subsection{Terapias antirretrovirais}

Entre 1987 e 1996 apenas as drogas antirretrovirais pertencentes à classe dos Inibidores da Transcriptase Reversa Análogos de Nucleosídeos (ITRNs) eram liberadas para uso clínico. Ainda no século XXI são as drogas mais usadas, pois formam a base de quase todos os esquemas antirretrovirais recomendados. Apresentam boa tolerância em curto prazo, no entanto, associam-se a sérios e irreversíveis efeitos colaterais após uso prolongado (RACHID et al, 2008). A zidovudina (AZT) foi o primeiro ITRNs a ser utilizado no tratamento em pessoas que vivem com HIV/Aids. Desta classe fazem parte também: Didanosina (ddl), Estavudina (d4T), Lamivudina (3TC), Abacavir (ABC), Emtricitabina (FTC) e Tenofovir (TDF) (FONSECA et al, 2012). 
A terapia antirretroviral (TARV) baseia-se na combinação de três drogas. Geralmente associam-se dois inibidores de transcriptase reversa análogos de nucleosídeos (NRTIs) a um inibidor de transcriptase reversa não-análogo de nucleosídeo (NNRTI) ou a um inibidor de protease (IP) (CAVENAGLI et al, 2012). Como regra, o esquema de primeira escolha adota as drogas Tenofovir (TDF), Lamivudina (3TC) e Efavirenz (EFV). A associação de tenofovir com lamivudina (TDF/3TC) apresenta um padrão de toxicidade melhor quanto a lipoatrofia e também aos hemocomponentes quando comparada ao AZT, e permite que seja tomada em dose única diariamente. No entanto, o tenofovir é um análogo de nucleotídeo (NRTIt) e apresenta como desvantagem a nefrotoxicidade principalmente em diabéticos, hipertensos, negros e idosos, além de estar associado também a diminuição da densidade óssea (MINISTÉRIO DA SAÚDE, 2015).

Por outro lado, o efavirenz, uma droga da classe dos NNRTI, apresenta um fator de supressão viral por longo prazo e possui um perfil de toxicidade favorável. Seus efeitos colaterais mais comuns - tonturas e alterações do sono - tendem a desaparecer após as primeiras quatro semanas do tratamento, embora sua prescrição deva ser criteriosamente avaliada em pessoas com depressão. Esquemas estruturados com NNRTI, particularmente com Efavirenz, possuem um padrão de toxicidade favorável, facilidade de administração posológica, maiores taxas de adesão ao tratamento, maior taxa de inibição da replicação viral e maior efetividade e durabilidade da supressão viral, se comparados com esquemas estruturados com inibidores de protease (MINISTÉRIO DA SAÚDE, 2015). Em casos especiais, em que haja necessidade de substituição do Efavirenz, a droga de escolha alternativa é a Nevirapina (NVP), que também pertence à classe dos NNRTI (RACHID et al, 2008).

Uma segunda linha de tratamento é adotada em situações em que o uso do Efavirenz e da Nevirapina seja contraindicado. Neste caso deve-se proceder à sua substituição por um IP, com escolha preferencial pelo Lopinavir com booster de Ritonavir (LPV/r) (MINISTÉRIO DA SAÚDE, 2014). Na falha virológica, os esquemas com IPs demonstram menor número de mutações de resistência do que os esquemas com NNRTI. Por outro lado, esta combinação apresenta mais frequentemente a ocorrência de eventos gastrointestinais e disfunções do metabolismo dos lipídeos e da glicose, quando comparados a esquemas que envolvem NNRTI (CAVENAGLI et al, 2012).

Fukumoto et al (2013) observou em seu estudo que esquemas terapêuticos contendo NNRTI foi mais frequentemente usado (81\%) em relação aos IP $(18,9 \%)$. A preferencia ao uso de NNRTI foi justificada pela presença de alterações metabólicas associadas ao uso de IP. Embora no mesmo estudo o autor não tenha encontrado diferenças significativas quando comparados os valores de linfócitos T CD4 dos pacientes que faziam uso de NNRTI aos dos pacientes em uso de IP.

A melhoria significativa da sobrevida de crianças, adolescentes e adultos que vivem com HIV em uso de TARV demonstra a eficácia das medidas terapêuticas adotadas até o momento (BAZIN et al, 2014). A adesão ao uso de medicamentos antirretrovirais em pessoas que vivem com HIV/Aids é um forte determinante para um tratamento promissor (ADEJUMO et al, 2015). 
As intervenções que melhoram a adesão ao tratamento estão associadas à supressão viral bem sucedida, redução dos riscos de infecções oportunistas e prevenção de resistência às drogas (UNAIDS, 2014). Enquanto que a não adesão ao tratamento está associada, em resumo, a redução da sobrevida das pessoas que vivem com HIV/Aids. De acordo com o Protocolo Clínico e Diretrizes Terapêuticas para Manejo da Infecção pelo HIV do Ministério da Saúde, o sucesso na adesão ao tratamento baseia-se na construção de componentes multifatoriais que envolvem aspectos físicos, psicológicos, sociais e culturais (MINISTÉRIO DA SAÚDE, 2015; TONNERA et al, 2015).

Segundo Adejumo (2015), a dificuldade de adesão ao tratamento está diretamente associada às condições sócio demográficas, a idade e aos efeitos secundários produzidos pelo uso das drogas. Além disto, a falência terapêutica pode ocorrer por diversos fatores e incluem complicações inadequadas, presença de co-morbidades, resistência viral prévia a um ou mais agentes antirretrovirais, absorção gastrointestinal alterada, interações entre drogas e um esquema terapêutico de baixa potência (FUKUMOTO et al, 2013).

Fonseca et al (2012) avaliou o esquema terapêutico de 229 pacientes e observou que $72,9 \%$ (167 pacientes) receberam um tratamento inapropriado, o que proporcionou um alto índice de abandono, motivados pelos efeitos indesejados ao organismo.

\section{ALTERAÇÕES ASSOCIADAS AO USO DAS TERAPIAS ANTIRRETROVIRAIS}

Embora o surgimento dos esquemas antirretrovirais represente um dos principais fatores responsáveis pela transformação da infecção pelo HIV, na condição de doença aguda para crônica, com um aumento considerável da qualidade e expectativa de vida, as consequências do tratamento em longo prazo têm sido abordadas em muitos estudos (ACOSTA et al, 2013; ADEJUMO et al, 2015; ALMEIDA et al, 2009; ARAÚJO et al, 2007; CARVALHO et al, 2014; CAVENAGLI, et al, 2012;; GUEVARA et al, 2008; KIBARU et al, 2015; LEITE et al, 2010; PAPI et al, 2014; PONTE et al, 2009; REMOR et al, 2002; SIGNORINI et al, 2010; VALENTE et al, 2005; VASIMON et al, 2011).

Desde o final da década de 90, estudos já apontam uma associação entre o uso da terapia antirretroviral e o aumento do risco de enfermidades coronárias (ACOSTA et al, 2013). No entanto, a ocorrência de anormalidades metabólicas crônicas como dislipidemias e lipodistrofias tornou-se mais evidente após a utilização dos inibidores de protease no esquema terapêutico, início da era HAART (ARAÚJO et al, 2007; CAVENAGLI et al, 2012).

Com a HAART novas complicações metabólicas emergiram. A lipodistrofia, caracterizada pela redistribuição da gordura corporal, é uma das alterações mais evidentes. A mais importante apresentação clínica desta síndrome é caracterizada pela perda de gordura (lipoatrofia), principalmente, na face, nos membros e nas nádegas e, em alguns casos, com acúmulo de gordura no abdômen, seios e região cervical (BARATA et al, 2013).

A Síndrome Metabólica (SM), caracterizada por um conjunto de fatores de riscos associados ao aumento da chance de desenvolvimento de doenças cardíacas, derrames e diabetes, tem sido constantemente identificada em indivíduos em uso de HAART (ACOSTA et al, 2013; ARAUJO et al, 2007; CAVENAGLI et al, 2012; TONNERA et al, 2015). A SM 
caracteriza-se pela redistribuição de gordura periférica, central ou visceral, associada à hiperglicemia, hipercolesterolemia, hipertrigliceridemia, hiperlipidemia, hipertensão arterial e resistência à insulina (CAVENAGLI et al, 2012).

Acosta et al (2003) realizou uma avaliação do perfil lipídico de uma determinada população com diagnóstico positivo para HIV e identificou um aumento significativo dos níveis de triglicerídeos entre os indivíduos que estavam em uso de HAART, sendo este resultado consistente com dados publicados em estudos com populações de outras regiões (ARAÚJO et al, 2007; CAVENAGLI et al, 2012). No entanto, em seu trabalho, foram encontrados resultados diferentes em relação a estudos anteriores quando comparado a prevalência da SM com fatores como gênero, idade e Índice de Massa Corporal (IMC).

Resultados semelhantes foram obtidos por Papi et al (2014). Seu estudo avaliou uma população de 90 crianças em uso de HAART e seus resultados mostram que mais da metade das crianças estudadas foram afetadas pela Síndrome da Lipodistrofia (SL). Destes casos, $22 \%$ apresentaram lipoatrofia e $32 \%$ lipohiperfrofia. Outro importante resultado, provavelmente associado com a resposta inflamatória, foi a relação entre os níveis de aminotransferase aspartato (AST) e a presença de SL, provavelmente justificada pela atividade inflamatória viral ou pelo acúmulo de gordura hepática.

Araujo (2007) desenvolveu um estudo com 372 pacientes em tratamento para HIV, a fim de comparar o esquema antirretroviral adotado e os efeitos secundários ao tratamento, bem como o status imunológico dos indivíduos, através da detecção dos níveis de carga viral e a contagem de linfócitos T CD4. Seus resultados mostraram que os pacientes com regimes antirretrovirais baseados em IP apresentaram maior probabilidade de desenvolver hipercolesterolemia e hipertrigliceridemia. Mas não encontrou diferença significativa quanto ao status imunológico ao comparar os diferentes regimes.

A terapia com drogas antirretrovirais pode conduzir também a uma alteração dos parâmetros hematológicos. Kibaru (2015) acompanhou crianças em tratamento de HAART por seis meses. Seus resultados sugerem que o uso da terapia induziu a mudanças hematológicas que podem se comportar de maneira benéfica ou maléfica. $\mathrm{O}$ autor sugere que o uso das terapias antirretrovirais, ao reduzir a carga viral, impede a instalação de infiltrados na medula óssea, promovendo uma melhoria dos parâmetros VCM (volume corpuscular médio) e HCM (hemoglobina corpuscular média), mas, por outro lado, o estudo demonstrou uma redução dos números totais de leucócitos e hemácias associadas ao uso das drogas.

Outro fator associado ao tratamento ou à própria infecção pelo HIV é a alteração do perfil hormonal. Diversas repercussões clínicas de disfunções hormonais elevam a morbimortalidade da infecção pelo HIV/Aids e reduzem a qualidade de vida das pessoas afetadas (PONTE et al, 2009). Já foi demonstrada a associação entre disfunção tireoidiana e a infecção pelo HIV, em combinação com o uso da HAART (CARVALHO et al, 2014). A presença de Doenças Tireoidianas Autoimunes foi observada em pacientes em uso de HAART com níveis de carga viral plasmática indetectável. Por outro lado, anormalidades no eixo gonadotrófico, em especial a redução das taxas de testosterona, são prevalentes em homens que vivem com HIV/Aids. Estudos iniciais evidenciaram hipogonadismo em aproximadamente 50\% dos homens com Aids (PONTE et al, 2009). 
Os ossos também fazem parte da lista dos componentes afetados pelos efeitos da terapia antirretroviral. A osteoporose, resultante da densidade óssea diminuída, tem sido identificada em pessoas que vivem com HIV/Aids independentemente de gênero e idade. Fatores como os efeitos das proteínas virais do HIV, a ação secundária dos esquemas de HAART e as citocinas inflamatórias têm sido associados a um padrão de baixa massa óssea (FERNÁNDEZ et al, 2014).

Um estudo comparando as alterações entre as densidades ósseas de indivíduos com HIV mostrou o impacto da HAART no metabolismo ósseo. Grund (2009) observou em seus resultados que pacientes em uso contínuo da terapia antirretroviral apresentaram $80 \%$ a mais de eventos de fratura óssea, quando comparados a pacientes que faziam uso intermitente do esquema terapêutico.

Contudo, evidências sugerem que a deterioração óssea relacionada ao HIV é provavelmente o resultado da adição de fatores de riscos tradicionais como uso de cigarro, álcool, deficiência de vitaminda $\mathrm{D}$, hipogonadismo, baixo peso corporal e fatores associados diretamente ao tratamento e infecção pelo HIV (FERNÁNDEZ et al, 2014).

Remor (2002) supõe ainda que o estado de saúde esteja modulando as distintas percepções sobre o tratamento, ou seja, aqueles que não experimentam uma evolução do tratamento tendem a decepcionar-se e desmotivar-se, o que gera imagens negativas e potencializa as dificuldades decorrentes da terapia. A associação destes fatores a uma avaliação frustrada contribui para o aumento dos níveis da ansiedade, repercutindo negativamente na qualidade do tratamento (SILVEIRA et al, 2012).

Já para Barata (2013), as manifestações físicas dos efeitos secundários ao tratamento antirretroviral, por exemplo, a lipodistrofia, afeta a autoestima que aumenta os sentimentos de estigma, o que pode causar mudanças no comportamento psicológico levando a uma participação limitada na vida social e profissional. Consequentemente, eventos de estresse, trauma e depressão produzem um efeito negativo em importantes componentes do sistema imune, como nos Linfócitos T CD4, que por sua vez, pode prejudicar a evolução do tratamento.

\section{CONSIDERAÇÕES FINAIS}

As intervenções farmacológicas representam a principal estratégia para garantir a qualidade e a expectativa de vida das pessoas que vivem com HIV/Aids. Entretanto, o uso dos antirretrovirais tem sido comumente associado a inúmeros efeitos adversos.

Dentre os efeitos mais comuns, destaca-se a redistribuição da gordura corporal associada à elevação dos níveis de colesterol, triglicerídeos e glicose. Outros estudos têm relacionado os antirretrovirais a alterações do eixo gonadotrófico, do metabolismo ósseo e da formação de hemocomponentes.

Sendo assim, a Aids deve ser encarada como uma doença que requer atenção, responsabilidades de assistência e cuidados específicos, seja das pessoas acometidas ou daquelas envolvidas diretamente com o seu tratamento. Os dados expostos no estudo, sugerem que o manejo adequado de pessoas que vivem com HIV/Aids requer uma rede 
profissional multidisciplinar composta por médicos, farmacêuticos, enfermeiros, biomédicos, nutricionistas, dentistas, psicólogos, assistentes sociais e gestores, todos devidamente capacitados e aptos a oferecer ao paciente as condições necessárias para o enfrentamento do tratamento que, embora com evolução crônica, requer muitas mudanças em seu padrão de vida.

\section{Association between the Use of Antiretroviral treatment for HIV and metabolic disorders}

\section{Abstract}

The use of antiretroviral therapies is essential to the life expectancy of those living with HIV / AIDS. However, studies show that the use of multiple drugs can induce side effects associated with metabolic disorders. Among the most common disorders are metabolic syndrome, which is characterized as a set of risk factors related to each other, with multifactorial pathogenesis and predisposing to the development of hypertension, diabetes mellitus and dyslipidemia. Changes in bone metabolism also represent an important event associated with treatment for HIV / AIDS. Studies showed that people using antiretroviral drugs were more likely to suffer fractures and also to develop osteoporosis. This review presents an update of the epidemiological and clinical factors of metabolic complications associated with the use of antiretroviral drugs in the course of HIV infection. However, AIDS must be seen as a disease that requires attention, care responsibilities and specific care, which requires a multidisciplinary professional network able to monitor and minimize the side effects associated with antiretroviral therapy.

Key-words: HIV, metabolic syndrome

\section{Referências}

ACOSTA, P. P.; UCRÓS, R. F.; BOVEA, A. S.; TRIANA, M. U.; LEÓN, I. Z.; GUSMÁN, N. A. Riesgo cardiovascular y síndrome metabólico en pacientes VIH positivos en el Caribe colombiano. Acta Med Colomb vol.38 no.4 Bogotá Oct./Dec. 2013

ADEJUMO, O. A.; MALEE, K. M.; RYSCAVAGE, P.; HUNTER, S. J.; TAIWO, O. B. Contemporary issues on the epidemiology and antiretroviral adherence of HIV- 
infected adolescents in sub-Saharan Africa: a narrative review. Journal of the Internacional AIDS Society. 2015

ALMEIDA, L. B.; GIUDICI, K. V.; JAIME, P. C.; Consumo alimentar e dislipidemia decorrente da terapia antirretroviral combinada para infecção pelo HIV: uma revisão sistemática. Arquivo Brasileiro de Endrocrinologia e Metabolismo. vol.53 no.5 São Paulo July. 2009

ARAÚJO, P. S. R.; XIMENES, R. A. A.; LOPES, C. F. C.; DUARTE, J. Y.; SILVA, M. M.; CARNEIRO, E. M. Antiretroviral treatment for HIV infections/aids and the risk of developing hyperglycemia and hyperlipidemia. Revista do Instituto de Medicina Tropical de São Paulo vol.49 no.2 São Paulo Mar./Apr. 2007

BARATA, A.; MALOUF, J.; GUTIERREZ, M.; MATEO, G. M.; SAMBEAT, M. A.; GICH, I.; CADAFALCH, J.; WULFF, J.; DOMINGO, P. Psychopathology and psychosocial adjustament in patients with HIV-associated lipodystroph. The Brazilian Journal of Infectious Diseases. Elsevier. Vol.17 no.4. 2013

BAZIN, G. R.; GASPAR, M. C. S.; SILVA, N. C. X. N.; MENDES, C. C.; OLIVEIRA, C. P.; BASTOS, L. S.; CARDOSO, C. A. Terapia antirretroviral em crianças e adolescentes infectados pelo HIV: o que sabemos após 30 anos de epidemia. Caderno de Saúde Pública. Vol.30 no.4. Rio de Janeiro. 2014

CARVALHO, E. G.; TEIXEIRA, P. F. S.; PANICO, A. B. G.; COHEN, M. V.; PINHEIRO, M. F. M. C.; BARROSO, P. F.; VAISMAN, M. Evaluation of thyroid function and autoimmunity in HIV-infected women. Arquivos Brasileiros de Endocrinologia e Metabologia. Vol.58.no.1.São Paulo.Fev.2014

CAVEnagli, F. M.; BAtaglion, C. A. N.; PAUlA, P. C.; MOTTA, A. C. F.; KOMESU, M. C.; Protease Inhibitor and Metabolic Alteration. International Journal Morphology. Vol.30.no.2. São Paulo. 2012

COFFIN, J.; HAASE, A.; LEVY, J. A.; MONTAGNIER, L.; OROSZLAN, S.; TEICH, N. What to call the AIDS vírus? Nature. Vol.10. 1986

FERNÁNDEZ, G. R.; GARCÍA, J. V.; PÉREZ, A. D.; ALHAMBRA, D. P. HIV infections, bone metabolismo, and fractures. Arquivo Brasileiro de Endocrionologia Metabólica. Vol.5. No.58. 2014

FONSECA, L. C.; MARTINS, F. J.; VIEIRA, R. C. P. A.; PEREIRA, R. M. C.; FERREIRA, A. S.; RAPOSO, N. R. P. Evaluation of inadequate anti-retroviral treatment in patients with HIV/AIDS. Revista da Sociedade Brasileira de Medicina Tropical. Vol.45. no2. 2012

FUKUMOTO, A. C. G.; OLIVEIRA, C. C.; TASKA, K. I.; SOUZA, L. R. Evolution of patients with AIDS after cART: clinical and laboratory evolution of patients with AIDS after 48 weeks of antirretroviral treatment. Revista Inst. Medicina Tropical. Vol.55. no.4. São Paulo. 2013 
GRUND, B.; PENG, G.; GILBERT, C. L.; HOY, J. F.; SHLAY, J. C. Continuous antiretroviral therapy decreases bone mineral density. AIDS. Vol.13 no.12. 2009

GUEVARA, F. O.; CAÑÓN, B.; LIÉVANO, M. C.; LOMBO, B.; RENDÓN, I.; BLANCO, F. Prevalencia de síndrome metabólico en pacientes infectados con VIH. Acta Med Colomb vol.33 no.4 Bogotá Oct./Dec. 2008

KIBARU, E. G.; NDUAT, R.; WAMALWA, D.; KARIUKI, N. Impact of highly active antiretroviral therapy on hematological indices among HIV- 1 infected children at Kenyatta National Hospital- Kenya: retrospective study. AIDS Research and therapy. 2015

LEITE, O. H. M. Alterações hematológicas associadas a infecção pelo HIV, ainda um problema? Rev. Bras. Hematol. Hemoter. vol.32 no.1 São Paulo Feb 2010

MINISTÉRIO DA SAÚDE. Boletim Epidemiológico HIV/Aids. Secretaria de Vigilância em Saúde. Departamento de DST, Aids e Hepatites Virais. Brasília. 2014

MINISTÉRIO DA SAÚDE. Protocolo Clínico e Diretrizes Terapêuticas para Manejo da Infecção pelo HIV em Adultos. Brasília. 2015.

PAPI, L.; MENEZES, A. C. G. B.; ROCHA, H.; ABREU, T. F.; OLIVEIRA, R. H.; FROTA, A. C. C.; EVAGELISTA, L. A.; HOFER, C. B. Prevalence of lipodystrophy and risk factors for dyslipidemia in HIV-infected children in Brazil. The Brazilian Journal of Infectious Diseases. Elsevier. Vol.18 no.04. 2014.

PONTE, M. M. C.; GURGEL, M. H. C.; MONTENEGRO, R. M. J. Disfunção do eixo gonadotrófico em homens com infecção pelo HIV/Aids. Arq Bras Endocrinol Metab. 2009

RACHID, M.; SCHECHTER, MAURO. Manual de HIV/AIDS. 9a Edição Revista e Ampliada. Editora Revinter. 2008

REMOR, E. A. Aspectos Psicossociais na Era dos Novos Tratamentos da AIDS. Psicologia: teoria e pesquisa. Vol.18.no.3.Brasilia. Sept/Dec. 2002

SIGNORINI, DE. J. H. P.; MONTEIRO, M. C. M.; SIGNORINI, D. H.; EYER-SILVA, W. A. Prevalence and determinant factors to lipid abnormalities among HIV-infected patients: a crosssectional study of 812 patients. Arquivo Brasileiro de Endrocrinologia e Metabolismo. vol.54 no.6 São Paulo Aug. 2010

SILVEIRA, M. P. T.; GUTTIER, M. C.; PINHEIRO, C. A. T.; PEREIRA, T. V. S.; CRUZEIRO, A. L. S.; MOREIRA, L. B. Depressive symptoms in HIV-infected patients treated with highly active antiretroviral therapy. Revista Brasileira de Psiquiatria. Vol.34. no.2. 2012

TONNERA, L. C. J.; MEIRELLES, B. H. S. Potencialidades e fragilidades da rede de cuidado da pessoa com HIV/Aids. Revista Brasileira de Enfermagem. Vol.68. no.3. 2015 
TSE, W. S.; YANG, W.; HUANG, W. A narrative review of cost-effectiveness analysis of people living with HIV treated with HAART: from interventions to outcomes. Clinico Economics and Outcome Research. Aug. 2015

UNAIDS. The gap report. Geneva, Switzerland: United Nations; 2014.

VALENTE, A. M. M.; REIS, A. F.; MACHADO, D. M.; SUCCI, R. C. M.; CHACRA, A. R. Alterações Metabólicas da Síndrome Lipodistrófica do HIV. Arquivo Brasileiro de Endrocrinologia e Metabolismo. vol.49.no.6. Dez 2005

VASIMON, H. S.; JORDAO, A. A.; PAULA, F. J. A.; MACHADO, A. A.; MONTEIRO, J. P. C Comparison of bioelectrical impedance with skinfold thickness and x-ray absorptiometry to measure body composition in HIV-infected with lipodistrophy. Nutr Hosp. 2011

WHO - WORD HEALTH ORGANIZATION. Validation of the Alcohol, Smoking and Substance Involvement Screnning Test (ASSIST) and Pilot Brief Intervention: A Technical Report of Phase II Finding of the WHO ASSIST Project. Management of Substance Abuse. Departament of Mental Health and Substance Abuse. 2006 


\section{AUTORIZAÇÃO PARA PUBLICAÇÃO DE TRABALHO}

Declaro para os devidos fins que o texto intitulado "Associação entre o uso de antirretrovirais no tratamento para HIV e alterações físicas e metabólicas" é de minha autoria e que todos os esforços foram feitos para que as fontes utilizadas no mesmo fossem explicitadas no próprio corpo do texto e nas referências. Estou ciente de que todas as informações apresentadas no trabalho são de exclusiva responsabilidade dos autores.

O presente trabalho: "Associação entre o uso de antirretrovirais no tratamento para HIV e alterações físicas e metabólicas" é inédito

Por meio desta declaração autorizo a divulgação do texto, por mídia impressa, eletrônica ou outra qualquer, sem custo algum. Esta obra passa a ser licenciada sob uma Licença Creative Commons Atribuicão 3.0.

Sem mais para o momento,

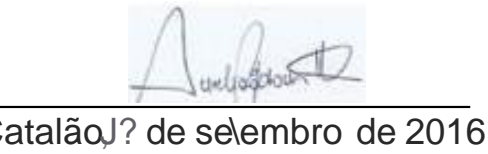

\begin{tabular}{|l|c|l|}
\hline \multicolumn{1}{|c|}{ Nome } & Assinatura & \multicolumn{1}{c|}{ E-mail } \\
\hline Aurélio Goulart Rodovalho & & aureliorodovalho@hotmail.com \\
\hline Franco Nero Tristão & - & franconeror-istao@bol.com br \\
\hline LailaL:dianeda Costa Galvão & $\mid$ & laila@iftm.edu br \\
\hline Ricardo Goulart Rodovalho & 5 & ricordogrodovolho@hotmoil.com \\
\hline Richelle Cristine Torunsky & - & richerct@gmail.com \\
\hline Roselma Lucchese & & roselmalucchese@hotmail.com \\
\hline
\end{tabular}

